\title{
Article \\ The Influence of the Implant Macrogeometry on Insertion Torque, Removal Torque, and Periotest Implant Primary Stability: A Mechanical Simulation on High-Density Artificial Bone
}

\author{
Margherita Tumedei ${ }^{1, *}$, Morena Petrini ${ }^{1}{ }^{\mathbb{D}}$, Davide Pietropaoli ${ }^{2} \mathbb{D}$, Alessandro Cipollina $^{3}$, Castrenze La Torre ${ }^{4}$, \\ Maria Stella Di Carmine ${ }^{5}$, Adriano Piattelli ${ }^{1,6,7,8, *}$ and Giovanna Iezzi ${ }^{1}$
}

check for updates

Citation: Tumedei, M.; Petrini, M.; Pietropaoli, D.; Cipollina, A.; La Torre, C.; Di Carmine, M.S.; Piattelli, A.; Iezzi, G. The Influence of the Implant Macrogeometry on Insertion Torque, Removal Torque, and Periotest Implant Primary Stability: A Mechanical Simulation on High-Density Artificial Bone. Symmetry 2021, 13, 776. https://doi.org/10.3390/sym13050776

Academic Editors: Carlos

Miguel Marto, Mafalda Laranjo and Ana Cristina Gonçalves

Received: 28 March 2021

Accepted: 20 April 2021

Published: 30 April 2021

Publisher's Note: MDPI stays neutral with regard to jurisdictional claims in published maps and institutional affiliations.

Copyright: (c) 2021 by the authors. Licensee MDPI, Basel, Switzerland. This article is an open access article distributed under the terms and conditions of the Creative Commons Attribution (CC BY) license (https:// creativecommons.org/licenses/by/ $4.0 /)$.
1 Department of Medical, Oral and Biotechnological Sciences, University “G. D'Annunzio" of Chieti Pescara, Via dei Vestini 31, 66100 Chieti, Italy; morena.petrini@unich.it (M.P.); gio.iezzi@unich.it (G.I.)

2 Department of Life, Health and Environmental Sciences, San Salvatore Hospital, University of L'Aquila-Dental Clinic_-Unit of Oral Diseases, Prevention and Translational Research-Oral Diseases and Systemic Interactions Study Group (ODISSY Group), 67100 L'Aquila, Italy; davide.pietropaoli@univaq.it Independent Researcher, Via Piacenza, 7, 92019 Sciacca, Italy; alexandros1960@libero.it

4 Independent Researcher, 91016 Erice, Italy; fabiolatorre78@gmail.com

5 Independent Researcher, Via Della Fornace n 10, 65014 Loreto Aprutino, Italy; mariastelladc@libero.it

6 Biomaterial Engineering, Catholic University of San Antonio de Murcia (UCAM), Av. de los Jerónimos, 135, 30107 Murcia, Spain

7 Villa Serena Foundation for Research, Via Leonardo Petruzzi 42, 65013 Città Sant'Angelo, Italy

8 Casa di Cura Villa Serena del Dott. L. Petruzzi, Via Leonardo Petruzzi 42, 65013 Città Sant'Angelo, Italy

* Correspondence: margherita.tumedei@unich.it (M.T.); apiattelli@unich.it (A.P.)

Abstract: Background: The primary stability is a determinant clinical condition for the success of different dental implants macro-design in different bone density using a validated and repeatable in vitro technique employing solid rigid polyurethane blocks. Materials and Methods: Five implants $3.8 \times 13 \mathrm{~mm}^{2}$ for each macro-design (i.e., IK—-tapered; IC—cylindric; and IA-active blade shape) were positioned into 20- and 30- pounds per cubic foot (PCF) polyurethane blocks. Bucco-lingual (BL) and mesial-distal (MD) implant stability quotient score (ISQ) was assessed by resonance frequency analysis while, insertion/removal torques were evaluated by dynamometric ratchet. Results: IC implants shown better primary stability in terms of ISQ compared to IA and IK in lower density block (20 PCF), while IK was superior to IA in higher density (30 PCF). IC shown higher removal torque in 30-PCF compared to IA and IC. Conclusions: The study effectiveness on polyurethane artificial bone with isotropic symmetry structure showed that the implants macro-design might represent a key factor on primary stability, in particular on low-density alveolar bone. Clinicians should consider patients features and implant geometry during low-density jaws rehabilitation. Further investigations are needed to generalize these findings.

Keywords: dental implant; primary stability; insertion torque; mechanical behaviour; drilling protocol

\section{Introduction}

Primary stability is the basic requirement to promote osseointegration and predictable long-term implant stability [1-3]. The parameters that primarily influence this factor are: bone quality, receiving site, preparation protocol, and macro and micro implant geometry $[4,5]$. Implant stabilization occurs by threads engaging the surrounding bone resulting in the transformation of a rotational force into a linear force. The stress generated at the bone-implant interface has three components: compressive, tensile, and shear forces [6]. Compressive forces directly influence the primary stability and within certain limits increase bone density [7]. Once this limit is exceeded, local ischemia and bone necrosis in the titanium-bone interface occur [8-10], whereas the tensile and shear forces 
are less beneficial. The free space created within the implant threads, resulting from the ratio of the drill/implant diameter, has been called the healing chamber [11]. The procedure for creating these healing chambers (overdrilling protocol) results in a significant decrease in the final insertion torque level in the implant [12]. The more abruptly the geometry increases, the more the surface of the thread penetrates or compresses the surrounding bone. The role of the thread as a retention element is related to a larger contact surface area, which provides greater bone-implant interaction and implies better primary stability, as demonstrated for immediate loading. In addition, good primary stability accelerates bone healing processes compared to implants with lower stability values.

Regardless low initial stability can allow micromovement of the implant during the healing period, and fibrous tissue can form at the interface between bone and implant and lead to failure [13]. In previous studies on thread configuration and force distribution in a static condition, implants with either squared or buttress threads reported that the axial load of these implants were mostly dissipated through a compressive force [14,15], whereas V-shaped and reverse buttress-threaded implants transmitted the axial force through a combination of compressive, tensile, and shear forces.

It is deduced that the face angle of the thread changes the direction of the force at the bone-implant interface $[15,16]$. In addition to the geometric design of the fixture, the thread pitch was also evaluated: the multiple configurations, which has two or more threads running parallel. These multiple thread implants allow a faster insertion, but according to a finite element analysis (FEA) study, the most favorable configuration in terms of implant stability was found to be the single thread, followed by the double one. The triple threaded implant was found to be the lower stable [17].

Polyurethane represents an excellent bio-simulator to mimic in vitro the behavior of implants with regard to parameters related to primary stability and stress under load [18]. The using of the polyurethane foam in form of solid rigid/composite has been considered The American Society for Testing and Materials indicated that the polyurethane foam in form of solid rigid/composite blocks and layers is the gold standard material to investigate the mechanical behavior and fatigue stress of dental implant device $[9,18] . I n$ fact, the polyurethane is provided by a constitutive isotropic symmetry and is able to simulate the mechanical response and densities of the human bone and easy handling using with linear elastic properties $[9,18]$. The aim of the present investigation was to compare the primary stability in vitro between three different dental implants, characterized by a different macro-design, positioned into two different densities of solid rigid polyurethane blocks.

\section{Materials and Methods}

\subsection{Implant General Characteristics}

A total of 30 implants $3.8 \mathrm{~mm}$ diameter and $13 \mathrm{~mm}$ length (Resista, Verbania, Italy), internal hexagon, were inserted on the polyurethane blocks (SawBones H, Pacific Research Laboratories Inc, Vashon, WA, USA), 5 screws for each experimental condition (Figure 1). 


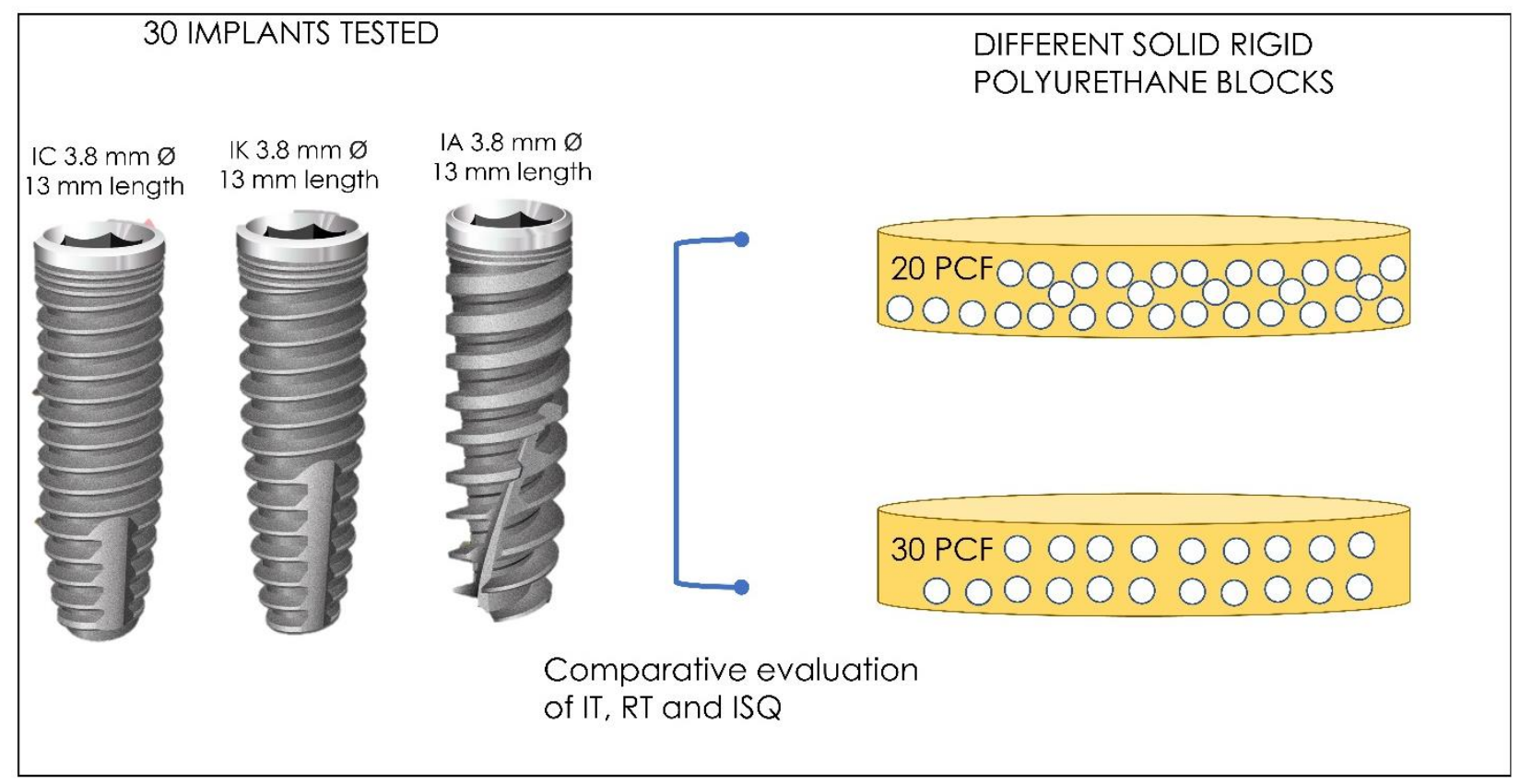

Figure 1. Graphical representation of the design of the study. PCF pounds per cubic foot block; IT insertion torque; RT removal torque; ISQ implant stability quotient score.

All implants tested were characterized by 4 different portions, starting from the implant neck:

1. Neck: smooth surface: machined, smoothed, rectify, and protected during the roughening process (neck portion).

2. Cylindric: nano-roughened surface: speedy treatment, nano-rough with average Ra less than 1 micron.

3. Screw pitch: micro-roughened surface 3/7: double etched (DAE) treatment, microrough with average Ra between 3 and $7 \mu \mathrm{m}$.

4. Tapered: micro-roughened surface 8/12: DAE treatment, micro-rough with average Ra between 8 and 12 micron.

The tested implants had 3 different macromorphologies (Figure 2):

(a) IK (tapered shape) (Resista, Verbania, Italy) are characterized by a smooth implant neck, $3.5 \mathrm{~mm}$ high, a rough cylindrical body (DAE differential micromorphology) with a screw pitch of $0.9 \mathrm{~mm}$ and a tapered apical portion, $6 \mathrm{~mm}$ long. The surface area developed for IK implants is $279.12 \mathrm{~mm}^{2}$.

(b) IC (cylindric shape) (Resista, Verbania, Italy) are characterized by a smooth implant neck $0.5 \mathrm{~mm}$ high, a rough cylindrical body (DAE differential micromorphology) with a screw pitch of $0.6 \mathrm{~mm}$ and a tapered apical portion, $3 \mathrm{~mm}$ long. The surface area developed for IC implants is $271.15 \mathrm{~mm}^{2}$.

(c) IA (active blade conic shape) (Resista, Verbania, Italy) are characterized by a smooth implant neck, $0.5 \mathrm{~mm}$ high, a rough cylindrical body (DAE differential micromorphology) with a screw pitch of $1.2 \mathrm{~mm}$ and a tapered apical portion $4 \mathrm{~mm}$ long. The surface area developed for IA implants is $280.57 \mathrm{~mm}^{2}$.

(d) All of implants tested presented a size of 3.8 diameter and $13 \mathrm{~mm}$ length. For each type, a total of 10 implants were used, 5 inserted in the polyurethane block 20 PCF and 5 inserted in the block 30 PCF (SawBones H, Pacific Research Laboratories Inc, Vashon, WA, USA). 


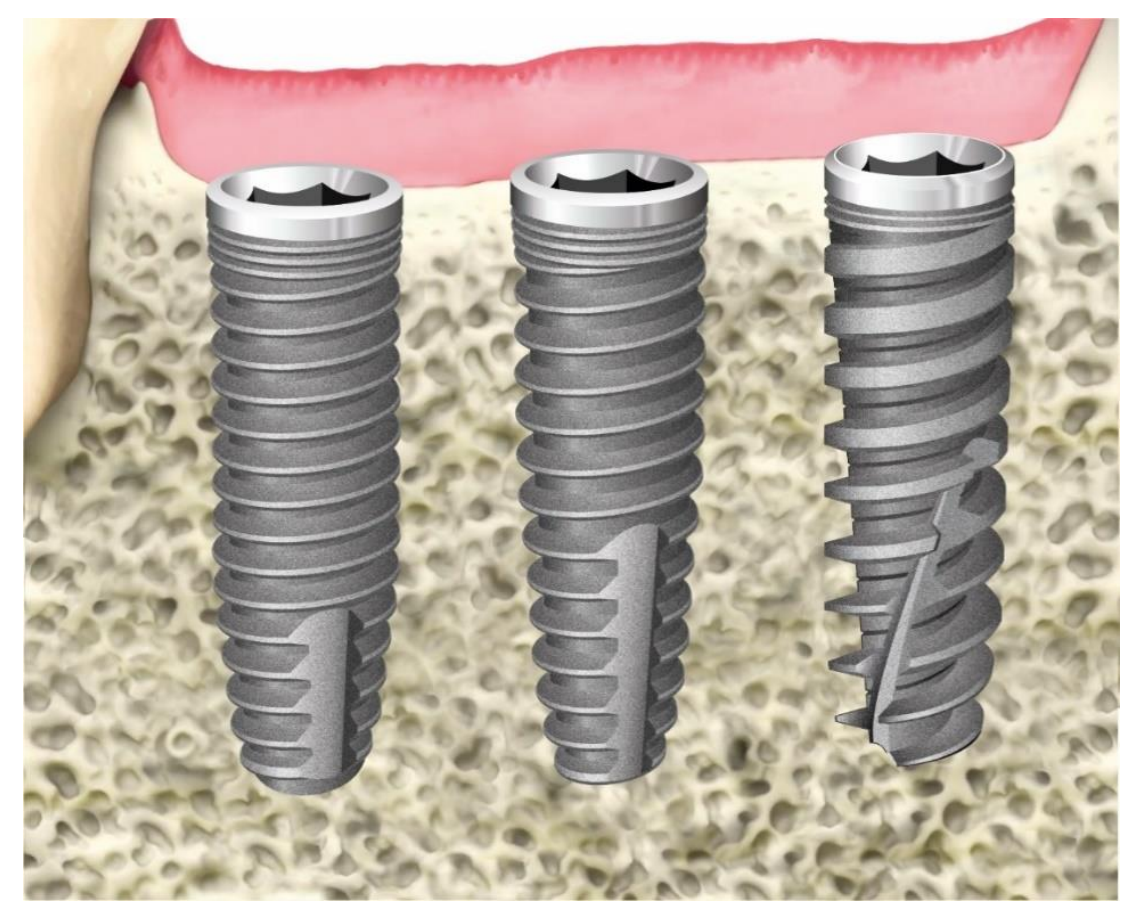

Figure 2. Graphical representation of the IC, IK, and IA implants.

\subsection{Polyurethane Foam Blocks}

A total of two different artificial bone in form of solid rigid polyurethane blocks (SawBones H, Pacific Research Laboratories Inc, Vashon, WA, USA) has been used for the present simulation. The material is homogeneous and it has been tested in different densities. The size of the polyurethane blocks used for the present investigation are $4.0 \mathrm{~cm} \times 13.0 \mathrm{~cm} \times 18.0 \mathrm{~cm}$. The experiment has been conducted according to a previously described methodology reported by Comuzzi et al., [19]. The 30 pounds per cubic foot block (PCF) simulated the D2 bone density, while the 20 PCF block simulated the D3 density according to the Misch classification [16].

\subsection{Drilling Protocol}

The drilling procedure has been performed according to the manufacturer protocol (Resista, Verbania, Italy). The surgical hand-piece was set with a speed of $600 \mathrm{rpm}$ and a torque of $30 \mathrm{~N}$ cm to perform the osteotomy.

The drills for IK (tapered shape) implants (nomenclature K) had 3 apical sections to adapt the implant site preparation to the geometry of implant core. The FI35 (Cortical Drill-Ø $3.5 \mathrm{~mm}$ ) was used for implants with $6.00 \mathrm{~mm}$ length.

The IK (tapered shape) implants were positioned according to the drill protocol: a pilot drill (FI 40 Ø $4.0 \mathrm{~mm}$ ), surgical twist drill C20 (FC 2018 Cylindrical Drill—Ø 2.0 mm-length $18 \mathrm{~mm}$ ), C26 (FC 2618 Cylindrical Drill—Ø $2.6 \mathrm{~mm}$-length $18 \mathrm{~mm}$ ), C32 (FC 3218 Cylindrical Drill—Ø $3.2 \mathrm{~mm}$-Long $18 \mathrm{~mm}$ ), and K38 (Final Drill—Ø $3.8 \mathrm{~mm}$-length $13 \mathrm{~mm}$ ).

The drills for IC (cylindric shape) implants had 2 apical sections to adapt the implant site preparation to the geometry of implant core. The IC (cylindric shape) and IA (active blade conic shape) implants were positioned following the same dedicated drilling protocol: a pilot drill (FI $40 \varnothing 4.0 \mathrm{~mm}$ ) for the cortical drill. A surgical twist drill C20 (FC 2018 Cylindrical Drill-Ø $2.0 \mathrm{~mm}$-length $18 \mathrm{~mm}$ ), C26 (FC 2618 Cylindrical Drill—Ø 2.6 $\mathrm{mm}$-length $18 \mathrm{~mm}$ ) and C32 (FC 3218 Cylindrical Drill—Ø $3.2 \mathrm{~mm}$-length $18 \mathrm{~mm}$ ). 


\subsection{Primary Stability Measurement}

Insertion and removal torque was measured using a dedicated dynamometric ratchet CRD (Resista, Verbania, Italy) (Figure 3).

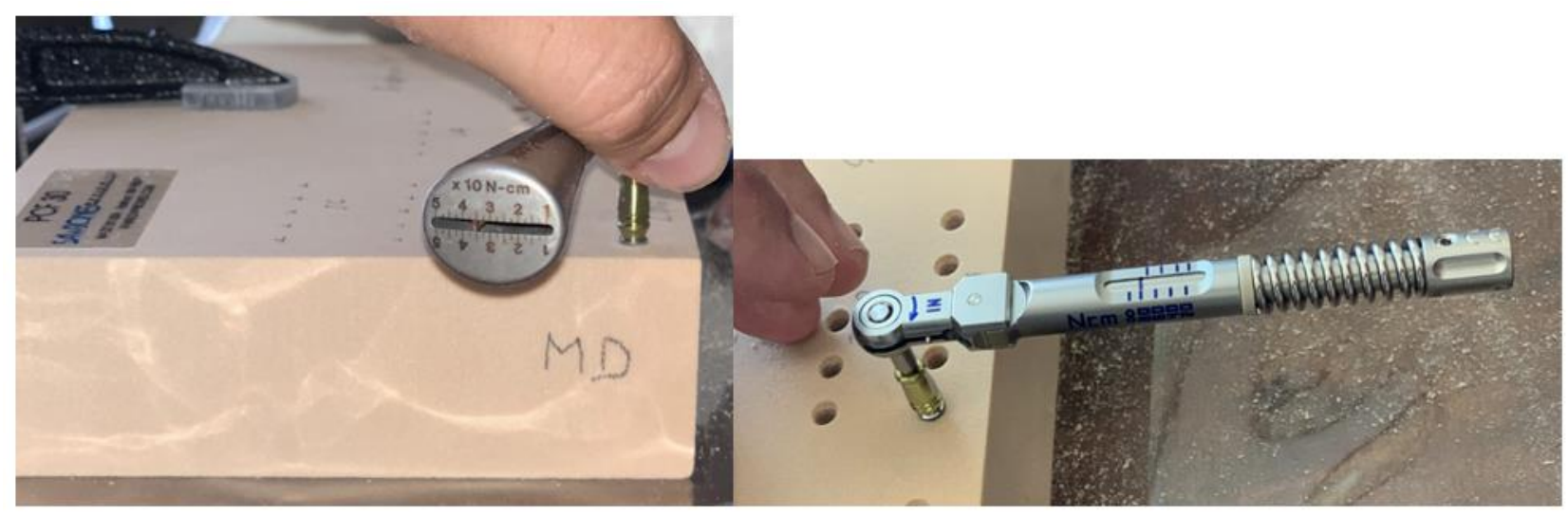

Figure 3. Details of the insertion and removal torque measured by the dedicated dynamometric ratchet.

The resonance frequency analysis (RFA) was measured through a dedicated electronic device (Osstel, Columbia, MD, USA) after the screw positioning. The measurements were classified according the implant stability quotient score (ISQ), ranged between 1 and 100 (low-good stability). The RFA was measured in two different points for each implant, which were perpendicular each other, in order to mimic the oral cavity:

- Mesio-distal

- Vestibulo-lingual

\subsection{Statistical Analysis}

Given the small sample size, we decided to apply a conservative approach based on nonparametric analyses. Multiple pairwise comparisons were evaluated by Wilcoxon test, while global $p$-value was tested with Kruskal-Wallis. Bonferroni correction for multiple comparison was applied as appropriate. Statistical significance was set at $p<0.05$. Data analyses and graphics were generated using $\mathrm{R}$ software (v.4.0.2). The sample size calculation was performed by the software package (Heinrich Heine Universität Düsseldorf, Germany) with 0.80 power (1- $\beta$ ), 0.05 alpha error and effect size (f) of 0.83 , according a previous study [20]. The minimum samples necessary to achieve the statistically significance of the study data was 30 implants (5 for each tested conditions).

\section{Results}

The Buccal-lingual (BL, Figure 4A) and mesial-distal (MD, Figure 1B) ISQ scores were globally similar for all the implant designs tested on 20-PCF blocks, while the same analysis on 30-PCF blocks showed a significant difference among implants designs (Figure 4A,B). Primary stability, in terms of ISQ score, was higher among implants placed in high density (i.e., 30-PCF) compared to low density (i.e., 20-PCF) polyurethane blocks. Pairwise comparisons showed that IC (cylindric shape) design was superior to IA (active blade conic shape) in terms of ISQ stability in all the scenarios, except for MD stability test on 30-PCF blocks (Figure 4A,B). Comparisons in the low-density setting demonstrated that (b) IC (cylindric shape) vs. IK (tapered shape) and IK vs. (c) IA (active blade conic shape) had similar ISQ stability, while in the high density setting there was evidence of ISQ superiority for the IK tapered shape design compared to the IA active blade conic shape design (Figure 4A,B). 

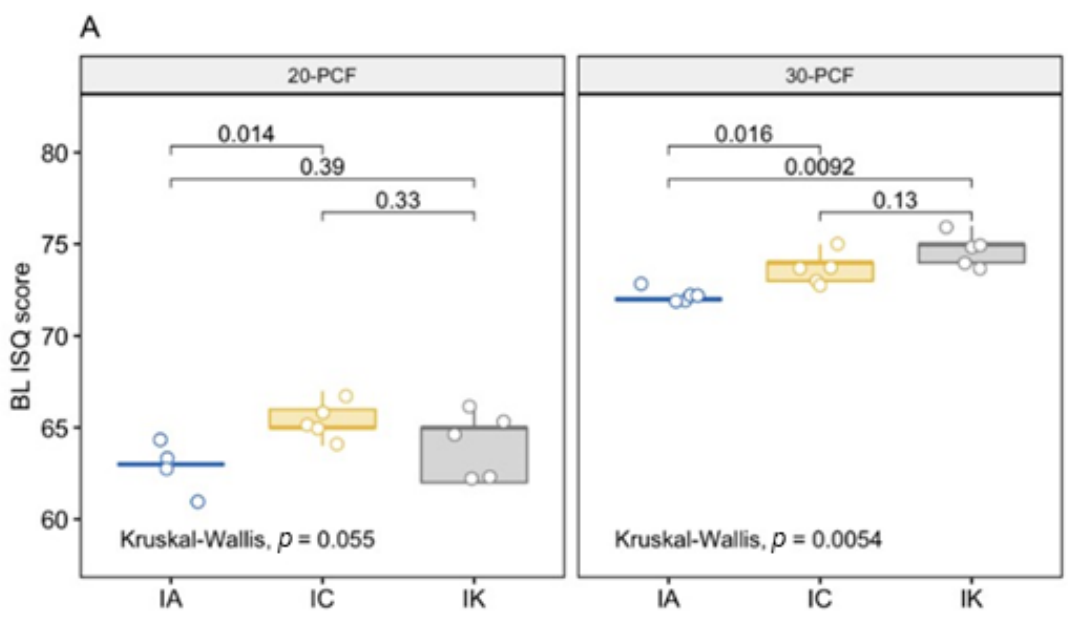

B
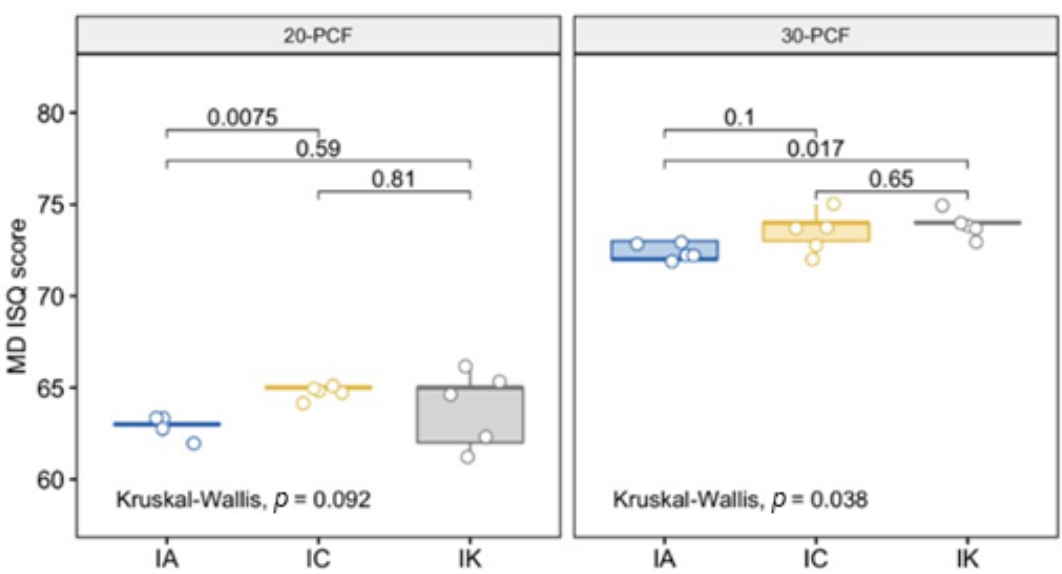

Figure 4. Charts representing the BL and MD ISQ, calculated for the study groups (Kruskal-Wallis test, $p<0.01$ ). (A) Buccal-Lingual ISQ score. (B) Mesial-Distal ISQ Score.

Torque analysis revealed that insertion and removal force were globally different across implants designs and experimental bone densities, except for insertion torque in 30-PCF blocks, where all dental implants needed the same insertion force in terms of $\mathrm{Ncm}$ (Figure 3). In line with this, less force was needed to place and remove implants in low density compared with high density blocks, irrespective of implant design (Figure 5A,B). Pairwise comparisons showed that the removal torque of IC (cylindric shape) and IK (tapered shape) was superior to IA in both densities, whereas that of IK (tapered shape) was similar to IC (cylindric shape) (Figure 5B). Intriguingly, the median insertion torque was the same in the high density setting irrespective of implants designs (Figure 5A), while IC (cylindric shape) showed the highest insertion torque compared with IA (active blade conic shape) design in 20-PCF blocks (Figure 5A). In particular, placing IC (cylindric shape) required a greater force compared with the IA (active blade conic shape) design, but a similar force compared with the IK (tapered shape) design. The IC (cylindric shape) design also required more insertion torque compared with the other design (Figure 5A). Means and standard deviations of the above-mentioned results were recapitulated in Table 1. 

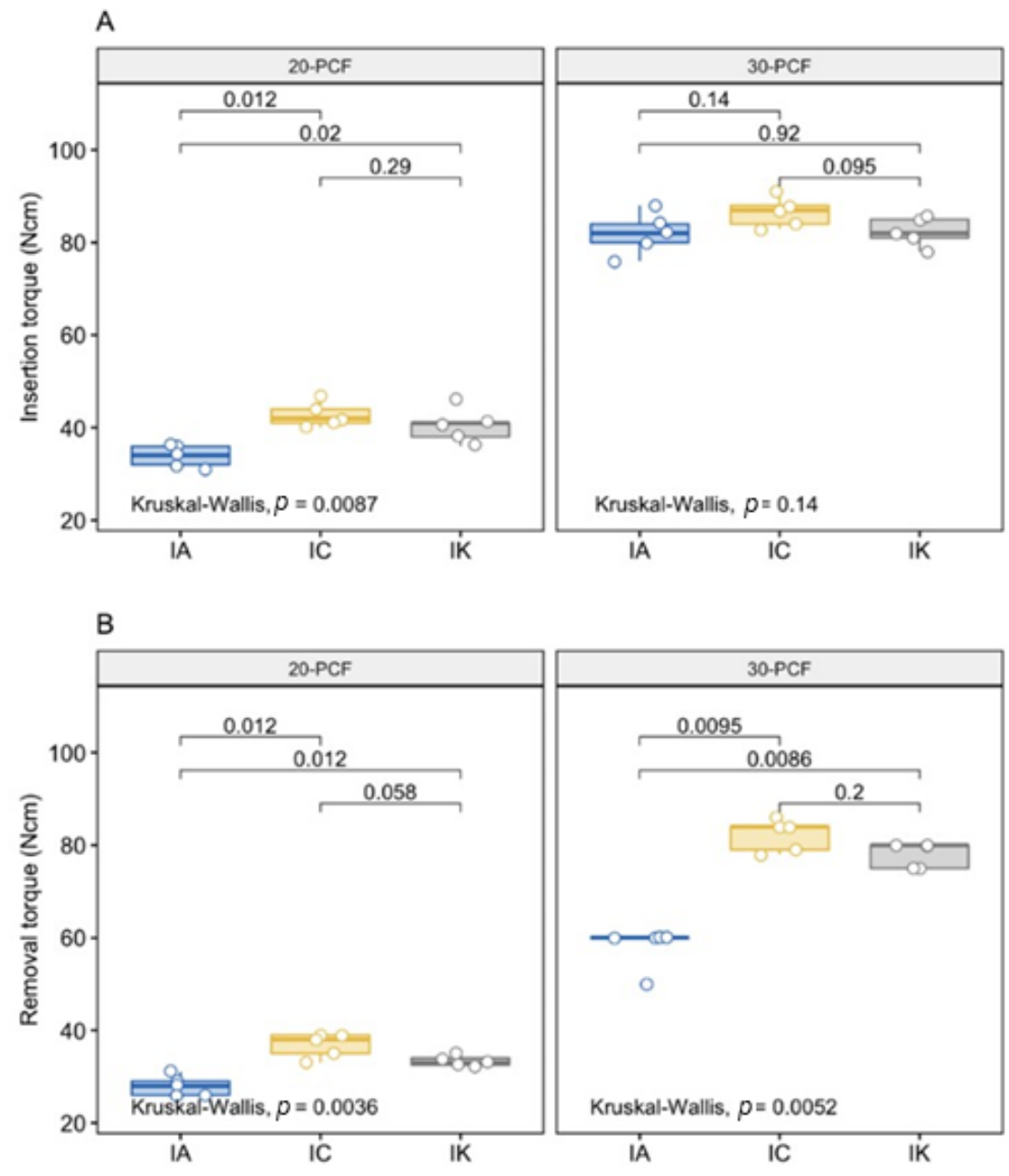

Figure 5. Charts representing the IT and RT, calculated for the study groups (Kruskal-Wallis test, $p<$ 0.01). (A) Insertion Torque of IA, IC, and IK implants. (B) Removal Torque of IA, IC, and IK implants.

Table 1. Summary of the descriptive statistical analysis of the BL and MD ISQ, IT, and RT of the study groups in different polyurethane densities.

\begin{tabular}{|c|c|c|c|c|c|c|c|}
\hline \multirow{2}{*}{ Implant Stability } & \multicolumn{3}{|c|}{ 20-PCF } & \multicolumn{3}{|c|}{ 30-PCF } & \multirow[t]{2}{*}{$p$-Value } \\
\hline & IA & IC & IK & IA & IC & IK & \\
\hline $\mathrm{n}$ & 5 & 5 & 5 & 5 & 5 & 5 & \\
\hline BL (mean (SD)) & $62.80(1.10)$ & $65.40(1.14)$ & $64.00(1.87)$ & $72.20(0.45)$ & $73.80(0.84)$ & $74.80(0.84)$ & $<0.001$ \\
\hline MD (mean (SD)) & $62.80(0.45)$ & $64.80(0.45)$ & $63.80(2.17)$ & $72.40(0.55)$ & $73.60(1.14)$ & $74.00(0.71)$ & $<0.001$ \\
\hline IT (mean (SD)) & $33.80(2.28)$ & $42.80(2.77)$ & $40.40(3.78)$ & $82.00(4.47)$ & $86.60(3.21)$ & $82.40(3.21)$ & $<0.001$ \\
\hline RT (mean (SD)) & $28.00(2.12)$ & $36.80(2.68)$ & $33.30(1.20)$ & $58.00(4.47)$ & $82.20(3.49)$ & $78.00(2.74)$ & $<0.001$ \\
\hline
\end{tabular}

\section{Discussion}

Our analysis shows that the IC (cylindric shape) design performed better compared to the IA (active blade conic shape) design in low-density (20-PCF) polyurethane blocks in terms of primary stability, insertion, and removal torque. The same comparisons on high-density (30-PCF) blocks indicated superiority of the cylindric shape over the others design in terms of stability scores, but not of insertion/removal torque.

Differences in implants geometry might explain these results in terms of mechanical stability, even though other uninvestigated factors might contribute to the relative findings. In particular, the IC cylindric shape design was characterized by $3 \mathrm{~mm}$ tapered apical 
portion, $0.9 \mathrm{~mm}$ screw pitch and $0.5 \mathrm{~mm}$ smooth neck, while the IA (active blade conic shape) design had a $4 \mathrm{~mm}$ tapered apical portion, $1.2 \mathrm{~mm}$ screw pitch and $0.5 \mathrm{~mm}$ smooth neck. The $1 \mathrm{~mm}$ difference between the tapered apical part of cylindric shape and active blade conic shape designs might translate into greater primary stability of the former in low density blocks in the experimental setting. Moreover, the cylindrical shape is clinically correlated to a higher insertion torque compared to conical screw, according to an increased interface contact area with the surrounding bone walls $[19,21]$. This evidence seems to disagree a part the literature that concern with others micro and nanotopographical characteristics plays important role for the achievement of implant primary. In fact, even though full tapered implants shown greater ISQ scores compared to the cylindrical design in less compact bone both in vitro [22] and in vivo [23], tapered geometry resulted in less percentage of bone-to-implant contact (BIC) compared with full cylindrical design [17]. In literature, the different pitch and their microscale irregularities has been reported as additive key-factor for implant stability in vivo [24]. In the present research, also the higher threads quantity, the different pitch and their microscale irregularities generated a significance different between the implant stability between the IA and IK conical implants, in both of cases considerable for immediate and delayed loading protocols. Thus, it might be possible that greater BIC of the cylindrical portion of IC cylindric shape compared to IA active blade conic shape $(10 \mathrm{~mm}$ vs. $9 \mathrm{~mm})$ contributes at least in part to an improvement in primary stability. This design characteristic is particularly useful in clinical situations where the achievement of primary stability is difficult to achieve due to sub-optimal bone properties, such as in post-extraction sites or in the case of low bone density [25]. From these basis, hybrid tapered-cylindric geometry represents a good compromise between BIC and post-surgical ISQ score [25].

Our findings can also result from other implant characteristics. Screw pitch, for example, represents another important factor for the stability of implants. Indeed, implants with smaller pitch might be beneficial for increasing immediate post-surgical ISQ score, especially in cancellous bone [17]. This is the case of the IC design that had a smaller pitch compared with the IA design $(0.9 \mathrm{~mm}$ vs. $1.2 \mathrm{~mm})$ and that performed better in the lowdensity scenario. An explanation could be that implants with lower pitch, and consequently more threads, have higher BIC [26] and stronger resistance to vertical load than those with greater pitch [27]. Furthermore, an accumulating body of evidence supports that greater BIC, obtained by reducing thread pitch, might improve initial anchorage and primary stability in cancellous bone [28]. In addition to clinical evidence, mathematical modelling implemented via finite element analysis (FEA) led to the same results, supporting the primary importance of thread pitch in implant stability [29]. However, the optimal pitch values vary in different thread shapes. In the FEA study, Lan and colleagues recommended $1.2 \mathrm{~mm}$ and $1.6 \mathrm{~mm}$ as the optimal thread pitches of triangular- and trapezoidal-threaded implants, respectively [30].

Another property that can translate into differences in stability is represented by neck height. The neck is a key element of tissue-bone-implant interface and related stability in the short- and the long-term [31]. Moreover, implants neck design may reduce both marginal bone loss and force stress over the surrounding structures [32]. It has been observed that the introduction of micro-rings on the implant neck may increase its stability, although no consensus has been reached regarding their specific features and designs [33]. Clinical evidence underlines the importance of implant neck as a notable factor for implant survival rate in the mid-term and the long- term [34].

While implants design is important for a long-lasting rehabilitation, patients' characteristics are the real keystone of a successful treatment. Recognized risk factors for implant loss, including, but not limited to periodontal diseases, diabetes, smoking habits, age, bruxism, and bone augmentation procedures, play a pivotal role in long-term implants' outcome [35,36]. Being poorly controllable over time, as they can worsen along with local or systemic health conditions, the management of such risk factors is the real challenge for clinicians planning implant-supported prosthesis. 
Our results are in line and expand current knowledge supporting the hypothesis that slightly differences in implant geometry might translate into visible long-lasting effects at the clinical level. In fact, several evidences implicated apical design, screw pitch, and neck characteristics as the determinant factors for a successful implant rehabilitation. Specifically, bone-implant interface, implants diameter and length, shape and characteristics of implant surface, prosthesis type, and the quantity and quality of the surrounding bone have an acknowledged importance for a successful treatment [37].

Our analysis on 20-PCF polyurethane blocks reproduces a critical clinical condition corresponding approximately to an in vivo D3 density, while the 30-PCF setting is more similar to the most common D2 bone jaws density [16]. Thus, a strength of this study is that the relative results might be translated to these real-world settings.

Our study has also limitations that deserve to be mentioned. The in vitro nature of the study does not necessarily reflect in vivo conditions, where multiple factors related to patients' characteristics contribute to determine implant survival in the short- and the long-term; and a single implant brand was tested, resulting in a weak generalizability of the relative findings.

\section{Conclusions}

Summarizing, our study provided an in vitro evidence that differences in implants geometry play a pivotal role in implant primary stability evaluated by RFA and insertion/removal torque, especially in the low-density scenario. Clinicians need to be aware of patients features and implant geometry, especially during the rehabilitation of lowdensity jaws.

Author Contributions: Conceptualization, A.P., G.I., and A.C.; methodology, A.P. and A.C.; software, M.T., M.P., and D.P.; validation, A.P. and G.I., investigation, M.T., M.P., A.C., C.L.T., and G.I.; writingoriginal draft preparation, D.P., M.T., M.P., and M.S.D.C.; writing—-review and editing, D.P., M.T., M.P., and M.S.D.C. All authors have read and agreed to the published version of the manuscript.

Funding: This research received no external funding.

Institutional Review Board Statement: Not applicable.

Informed Consent Statement: Not applicable.

Data Availability Statement: All experimental data to support the findings of this study are available contacting the corresponding author upon request.

Acknowledgments: The authors declare no acknowledgment for the present research.

Conflicts of Interest: The authors declare no conflict of interest.

\section{References}

1. Degidi, M.; Piattelli, A.; Gehrke, P.; Felice, P.; Carinci, F. Five-year outcome of 111 immediate nonfunctional single restorations. J. Oral Implantol. 2006, 32, 277-285. [CrossRef] [PubMed]

2. Scarano, A.; de Oliveira, P.S.; Traini, T.; Lorusso, F. Sinus Membrane Elevation with Heterologous Cortical Lamina: A Randomized Study of a New Surgical Technique for Maxillary Sinus Floor Augmentation without Bone Graft. Materials 2018, 11 , 1457. [CrossRef] [PubMed]

3. Scarano, A.; Murmura, G.; Vantaggiato, G.; Lauritano, D.; Silvestre-Rangil, J.; DI Cerbo, A.; Lorusso, F. Delayed Expansion of Atrophic Mandible (Deam): A Case Report. Oral Implantol. 2017, 10, 190-196. [CrossRef]

4. Matys, J.; Świder, K.; Flieger, R.; Dominiak, M. Assessment of the Primary Stability of Root Analog Zirconia Implants Designed Using Cone Beam Computed Tomography Software by Means of the Periotest ${ }^{\circledR}$ Device: An Ex Vivo Study. A Preliminary Report. Adv. Clin. Exp. Med. 2017, 26, 803-809. [CrossRef] [PubMed]

5. Amid, R.; Raoofi, S.; Kadkhodazadeh, M.; Movahhedi, M.R.; Khademi, M. Effect of Microthread Design of Dental Implants on Stress and Strain Patterns: A Three-Dimensional Finite Element Analysis. Biomed. Tech. Biomed. Eng. 2013, 58. [CrossRef]

6. Udomsawat, C.; Rungsiyakull, P.; Rungsiyakull, C.; Khongkhunthian, P. Comparative Study of Stress Characteristics in Surrounding Bone during Insertion of Dental Implants of Three Different Thread Designs: A Three-Dimensional Dynamic Finite Element Study. Clin. Exp. Dent. Res. 2019, 5, 26-37. [CrossRef] [PubMed] 
7. Tumedei, M.; Piattelli, A.; Degidi, M.; Mangano, C.; Iezzi, G. A Narrative Review of the Histological and Histomorphometrical Evaluation of the Peri-Implant Bone in Loaded and Unloaded Dental Implants. A 30-Year Experience (1988-2018). Int. J. Environ. Res. Public Health 2020, 17, 2088. [CrossRef] [PubMed]

8. Nedir, R.; Bischof, M.; Szmukler-Moncler, S.; Bernard, J.-P.; Samson, J. Predicting Osseointegration by Means of Implant Primary Stability. A Resonance-Frequency Analysis Study with Delayed and Immediately Loaded ITI SLA Implants. Clin. Oral Implant. Res. 2004, 15, 520-528. [CrossRef] [PubMed]

9. Isoda, K.; Ayukawa, Y.; Tsukiyama, Y.; Sogo, M.; Matsushita, Y.; Koyano, K. Relationship between the Bone Density Estimated by Cone-Beam Computed Tomography and the Primary Stability of Dental Implants: Relationship between Bone Density and Implant Stability. Clin. Oral Implant. Res. 2012, 23, 832-836. [CrossRef]

10. Bilhan, H.; Geckili, O.; Mumcu, E.; Bozdag, E.; Sünbüloğlu, E.; Kutay, O. Influence of Surgical Technique, Implant Shape and Diameter on the Primary Stability in Cancellous Bone: What influences primary stability in cancellous bone. J. Oral Rehabil. 2010, 37, 900-907. [CrossRef]

11. Del Giudice, R.; Piattelli, A.; Grande, N.-M.; Cataneo, E.; Crispino, A.; Petrini, M. Implant Insertion Torque Value in Immediate Loading: A Retrospective Study. Med. Oral Patol. Oral Cir. Bucal 2019, 24, e398-e403. [CrossRef] [PubMed]

12. Kotsu, M.; Urbizo Velez, J.; Bengazi, F.; Tumedei, M.; Fujiwara, S.; Kato, S.; Botticelli, D. Healing at Implants Installed from 70to <10-Ncm Insertion Torques: An Experimental Study in Dogs. Oral Maxillofac. Surg. 2020. [CrossRef]

13. Javed, F.; Romanos, G.E. The Role of Primary Stability for Successful Immediate Loading of Dental Implants. A Literature Review. J. Dent. 2010, 38, 612-620. [CrossRef]

14. Hansson, S.; Werke, M. The Implant Thread as a Retention Element in Cortical Bone: The Effect of Thread Size and Thread Profile: A Finite Element Study. J. Biomech. 2003, 36, 1247-1258. [CrossRef]

15. Misch, C.E.; Strong, I.T.; Bidez, M.W. Implant Design. In Contemporary Implant Dentistry; Mosby Inc.: Mariland Heights, MO, USA, 2007; p. 200.

16. Misch, C.E. Bone Density: A Key Determinant for Treatment Planning. In Contemporary Implant Dentistry, 3rd ed.; Mosby: St. Louis, MO, USA, 2007; pp. 130-146.

17. Abuhussein, H.; Pagni, G.; Rebaudi, A.; Wang, H.-L. The Effect of Thread Pattern upon Implant Osseointegration. Clin. Oral Implant. Res. 2010, 21, 129-136. [CrossRef] [PubMed]

18. Fanali, S.; Tumedei, M.; Pignatelli, P.; Inchingolo, F.; Pennacchietti, P.; Pace, G.; Piattelli, A. Implant Primary Stability with an Osteocondensation Drilling Protocol in Different Density Polyurethane Blocks. Comput. Methods Biomech. Biomed. Eng. 2020, 1-7. [CrossRef] [PubMed]

19. Comuzzi, L.; Tumedei, M.; Pontes, A.E.; Piattelli, A.; Iezzi, G. Primary Stability of Dental Implants in Low-Density (10 and 20 Pcf) Polyurethane Foam Blocks: Conical vs. Cylindrical Implants. Int. J. Environ. Res. Public Health 2020, 17, 2617. [CrossRef] [PubMed]

20. Tumedei, M.; Petrini, M.; Cipollina, A.; Di Carmine, M.; Piattelli, A.; Cucurullo, A.; Iezzi, G. Comparative Evaluation of Primary Stability between Different Diameters Multi-Scale Roughness Dental Implant by Solid Rigid Polyurethane Simulation. Osteology 2021, 1, 62-72. [CrossRef]

21. Staedt, H.; Palarie, V.; Staedt, A.; Wolf, J.M.; Lehmann, K.M.; Ottl, P.; Kämmerer, P.W. Primary Stability of Cylindrical and Conical Dental Implants in Relation to Insertion Torque-A Comparative Ex Vivo Evaluation. Implant. Dent. 2017, 26, 250-255. [CrossRef]

22. Menicucci, G.; Pachie, E.; Lorenzetti, M.; Migliaretti, G.; Carossa, S. Comparison of Primary Stability of Straight-Walled and Tapered Implants Using an Insertion Torque Device. Int. J. Prosthodont. 2012, 25, 465-471.

23. Atieh, M.A.; Alsabeeha, N.; Duncan, W.J. Stability of Tapered and Parallel-Walled Dental Implants: A Systematic Review and Meta-Analysis. Clin. Implant. Dent. Relat. Res. 2018, 20, 634-645. [CrossRef]

24. Meirelles, L.; Currie, F.; Jacobsson, M.; Albrektsson, T.; Wennerberg, A. The Effect of Chemical and Nanotopographical Modifications on the Early Stages of Osseointegration. Int. J. Oral Maxillofac. Implant. 2008, 23, 641-647.

25. Alves, C.C.; Neves, M. Tapered Implants: From Indications to Advantages. Int. J. Periodontics Restor. Dent. 2009, $29,161-167$.

26. Roberts, W.E.; Smith, R.K.; Zilberman, Y.; Mozsary, P.G.; Smith, R.S. Osseous Adaptation to Continuous Loading of Rigid Endosseous Implants. Am. J. Orthod. 1984, 86, 95-111. [CrossRef]

27. Ma, P.; Xiong, W.; Tan, B.; Geng, W.; Liu, J.; Li, W.; Li, D. Influence of Thread Pitch, Helix Angle, and Compactness on Micromotion of Immediately Loaded Implants in Three Types of Bone Quality: A Three-Dimensional Finite Element Analysis. BioMed Res. Int. 2014, 2014, 983103. [CrossRef] [PubMed]

28. Ryu, H.-S.; Namgung, C.; Lee, J.-H.; Lim, Y.-J. The Influence of Thread Geometry on Implant Osseointegration under Immediate Loading: A Literature Review. J. Adv. Prosthodont. 2014, 6, 547-554. [CrossRef]

29. Chang, P.-K.; Chen, Y.-C.; Huang, C.-C.; Lu, W.-H.; Chen, Y.-C.; Tsai, H.-H. Distribution of Micromotion in Implants and Alveolar Bone with Different Thread Profiles in Immediate Loading: A Finite Element Study. Int. J. Oral Maxillofac. Implant. 2012, 27, e96-e101.

30. Lan, T.-H.; Du, J.-K.; Pan, C.-Y.; Lee, H.-E.; Chung, W.-H. Biomechanical Analysis of Alveolar Bone Stress around Implants with Different Thread Designs and Pitches in the Mandibular Molar Area. Clin. Oral Investig. 2012, 16, 363-369. [CrossRef]

31. Hansson, S. The Implant Neck: Smooth or Provided with Retention Elements. A Biomechanical Approach. Clin. Oral Implant. Res. 1999, 10, 394-405. [CrossRef] 
32. Koodaryan, R.; Hafezeqoran, A. Evaluation of Implant Collar Surfaces for Marginal Bone Loss: A Systematic Review and Meta-Analysis. BioMed Res. Int. 2016, 2016, 4987526. [CrossRef]

33. Calvo-Guirado, J.L.; Jiménez-Soto, R.; Pérez Albacete-Martínez, C.; Fernández-Domínguez, M.; Gehrke, S.A.; Maté-Sánchez de Val, J.E. Influence of Implant Neck Design on Peri-Implant Tissue Dimensions: A Comparative Study in Dogs. Materials 2018, 11, 2007. [CrossRef] [PubMed]

34. Spies, B.C.; Bateli, M.; Ben Rahal, G.; Christmann, M.; Vach, K.; Kohal, R.-J. Does Oral Implant Design Affect Marginal Bone Loss? Results of a Parallel-Group Randomized Controlled Equivalence Trial. BioMed Res. Int. 2018, 2018. [CrossRef]

35. Dreyer, H.; Grischke, J.; Tiede, C.; Eberhard, J.; Schweitzer, A.; Toikkanen, S.E.; Glöckner, S.; Krause, G.; Stiesch, M. Epidemiology and Risk Factors of Peri-Implantitis: A Systematic Review. J. Periodontal Res. 2018, 53, 657-681. [CrossRef] [PubMed]

36. Schimmel, M.; Srinivasan, M.; McKenna, G.; Müller, F. Effect of Advanced Age and/or Systemic Medical Conditions on Dental Implant Survival: A Systematic Review and Meta-Analysis. Clin. Oral Implant. Res. 2018, 29, 311-330. [CrossRef] [PubMed]

37. Ding, X.; Liao, S.-H.; Zhu, X.-H.; Zhang, X.-H.; Zhang, L. Effect of Diameter and Length on Stress Distribution of the Alveolar Crest around Immediate Loading Implants. Clin. Implant. Dent. Relat. Res. 2009, 11, 279-287. [CrossRef] 